\section{WHITEN BY DAY OR BY NIGHT}

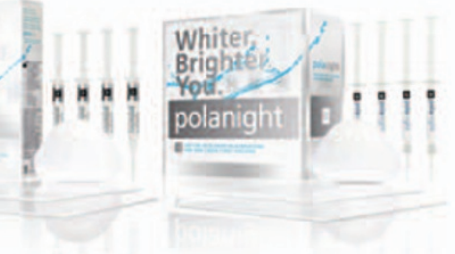

Poladay and Polanight from

Southern Dental Industries (SDI) are two cosmetic tooth whitening systems that will help your patients achieve a whiter and healthier smile.

Both Poladay and Polanight:

- Are pH neutral which ensures the full release of the peroxide without patient discomfort

- Have a high water content which reduces dehydration

- Contain desensitising agents

- Have a long lasting fresh spearmint taste

- Incorporate special additives that minimise plaque formation.

This makes both systems mild enough to ensure complete comfort throughout use.

The main difference between Poladay and Polanight lies in the active ingredients that are used to bleach the teeth. Poladay uses hydrogen peroxide which is faster working, for a quicker treatment. Polanight, however, uses carbamide peroxide, a slower acting chemical that will provide a gentler whitening process.

Poladay trays can be worn for as little as 30 minutes a day and repeated for 5-10 days depending on requirements. Polanight can be worn for as little as 40 minutes a day or overnight.

The Dental Directory is offering its customers a free box of SDI's Poladay or Polanight for every three boxes ordered.

For more information, contact The Dental Directory on 0800 585 586, or visit www.dentaldirectory.co.uk.

\section{FOR ALL YOUR RESTORATIVE NEEDS}

Plandent stock a huge range of dental restorative materials for you to choose from. Whether you are looking for one of the latest, technologically advanced composite materials on the market or the amalgam that you have been using for some time: look no further.

Plandent keep in stock a full range of the leading brands of restorative dental materials: glass ionomers, compomers, composites, temporary cements or any of the accessories that you use on a daily basis. Many of these restorative materials are regularly promoted at extra special prices.

Due to the group buying power of Plandent and efficient supply chain, Plandent offer price reductions against recommended dental prices, meaning a great deal for you.

Whether you're looking for dental consumables, a new handpiece or capital equipment, Plandent offer you everything you could possibly need for your surgery. All equipment sales are fully backed by Plandent's comprehensive after sales customer care programme.

For further information call free on 0500500322.

\title{
CURING IN SEVEN VIBRANT COLOURS
}

General Medical now offer the complete range of Mectron LED Curing Lights in a wide range of exciting colours and finishes. They offer exceptional value for money and the quality you would expect from one of the world's leading manufacturers.

Well balanced for optimised user comfort and weighing just $105 \mathrm{~g}$, the Mectron Starlight Pro cordless curing light offers a choice of 'fast' 10 second or 'slow rise' 20 second curing modes; more than $1000 \mathrm{~mW} / \mathrm{cm}^{2}$ output; a compact base unit featuring an integrated radiometer to check output and green and red lights to indicate battery status; a trickle-charge lithium-ion battery which delivers at least 320 cycles of $100 \%$ consistent output per single charge, free from memory effect; acoustic low battery warning; and a choice of seven vibrant colours. Featuring the latest LED technology Mectron Starlight Pro is silent, does not require a fan and delivers on average 2.5 million 10 second cycles. General Medical also offer the Mectron Starlight Pro Sport with a bright red, matt black or shiny black finish, 10\% more power and all the other advantages of the Mectron Starlight Pro.

For further information call 01380734990 or visit www.generalmedical.co.uk.

\section{GIVE YOUR PATIENTS A SKIN SELECTION BOX}

OXYjet UK offer a comprehensive range of skin care products which will enable you to enhance your patients' sense of well-being and boost your income.

The Beauty-Tox range contains an exclusive cocktail of natural ingredients that help eliminate the signs of ageing. These ingredients are combined together in various formulations, available separately or as part of the attractive Beauty-Tox Selection Box.

The INtact range has been specifically created with the slimmer woman in mind because they are generally the first to show the signs of ageing. INtact replenishes the skin's natural deposits and provides more structure and volume leaving the skin with a fresher, younger appearance.

Competitively priced, you can generate a 35\% profit on every OXYjet skin care product you sell. OXYjet UK even provide free of charge point-ofsales support materials to help generate business and there is no minimum order quantity.

Telephone 01775 722243, email enquiries@oxyjetuk.co.uk or visit www.oxyjetuk.co.uk. 